\title{
Changes in cerebral metabolism in patients with a minimally conscious state responding to zolpidem
}

\author{
Camille Chatelle ${ }^{1,2+}$, Aurore Thibaut ${ }^{1+}$, Olivia Gosseries ${ }^{1,3}$, Marie-Aurélie Bruno ${ }^{1}$, Athena Demertzi ${ }^{1}$, \\ Claire Bernard ${ }^{4}$, Roland Hustinx ${ }^{4}$, Luaba Tshibanda ${ }^{5}$, Mohamed A. Bahri ${ }^{1}$ and Steven Laureys ${ }^{1 *}$ \\ ${ }^{1}$ Coma Science Group, Cyclotron Research Centre, University and University Hospital of Liège, Liège, Belgium \\ ${ }^{2}$ Neurorehabilitation Lab, Department of Physical Medicine and Rehabilitation, Spaulding Rehabilitation Hospital, Harvard Medical School, Boston, MA, USA \\ ${ }^{3}$ Center for Sleep and Consciousness, and Postle Laboratory, Department of Psychiatry, University of Wisconsin, Madison, WI, USA \\ ${ }^{4}$ Nuclear Medicine Department, University Hospital of Liège, Liège, Belgium \\ ${ }^{5}$ Department of Neuroradiology, University Hospital of Liège, Liège, Belgium
}

\section{Edited by:}

Marta Olivetti, Sapienza University

of Rome, Italy

Reviewed by:

Andrew Michael Goldfine, Stony

Brook University, USA

Francesca Pistoia, University of

L'Aquila, Italy

\section{${ }^{*}$ Correspondence:}

Steven Laureys, Coma Science

Group, University Hospital of Liège,

Sart Tilman B35, 4000-Liège,

Belgium

e-mail: steven.laureys@ulg.ac.be

${ }^{\dagger}$ These authors have contributed equally to this work.

\begin{abstract}
Background: Zolpidem, a short-acting non-benzodiazepine GABA agonist hypnotic, has been shown to induce paradoxical responses in some patients with disorders of consciousness (DOC), leading to recovery of arousal and cognitive abilities. We here assessed zolpidem-induced changes in regional brain metabolism in three patients with known zolpidem response in chronic post-anoxic minimally conscious state (MCS).
\end{abstract}

Methods: [18F]-fluorodeoxyglucose positron emission tomography (FDG-PET) and standardized clinical assessments using the Coma Recovery Scale-Revised were performed after administration of $10 \mathrm{mg}$ zolpidem or placebo in a randomized double blind 2-day protocol. PET data preprocessing and comparison with a healthy age-matched control group were performed using statistical parametric mapping (SPM8).

Results: Behaviorally, all patients recovered functional communication after administration of zolpidem (i.e., emergence from the MCS). FDG-PET showed increased metabolism in dorsolateral prefrontal and mesiofrontal cortices after zolpidem but not after placebo administration.

Conclusion: Our data show a metabolic activation of prefrontal areas, corroborating the proposed mesocircuit hypothesis to explain the paradoxical effect of zolpidem observed in some patients with DOC. It also suggests the key role of the prefrontal cortices in the recovery of functional communication and object use in hypoxic patients with chronic MCS.

Keywords: minimally conscious state, zolpidem, brain metabolism, positron emission tomography, prefrontal cortex, mesocircuit hypothesis

\section{INTRODUCTION}

Following a severe brain injury, patients can stay in a prolonged period of unconsciousness. They can be in a coma for few days or weeks, before evolving into a vegetative/unresponsive wakefulness syndrome (i.e., eyes open but only showing reflex behaviors Laureys et al., 2010) or into a minimally conscious state (MCS, Giacino et al., 2002). MCS is characterized by inconsistent but reproducible evidence of awareness (e.g., signs of consciousness without command following- MCS minus; with command following- MCS plus, Bruno et al., 2011). Emergence from MCS (EMCS) is characterized by the recovery of functional communication and/or functional object use. According to the Coma Recovery Scale-Revised assessment (CRS-R, Giacino et al., 2004), functional communication is reached when a patient is able to answer 6 out of 6 "yes-no" questions accurately. Communication is intentional if (s)he is able to answer at least 2 out of 6 questions independently of accuracy. Functional object use encompasses the ability to accurately use two different objects two times. Research on efficient treatments improving cognitive abilities in this population of patients with MCS has shown that deep brain stimulation (Schiff et al., 2007) and some pharmacological agents such as amantadine (Schnakers et al., 2008a; Giacino et al., 2012), apomorphine (Fridman et al., 2009) intrathecal baclofen (Sara et al., 2007) and zolpidem (Whyte and Myers, 2009; Thonnard et al., 2013) can improve recovery in some cases (for a recent review, see Gosseries et al., 2013). However, the mechanisms underlying these recoveries remain poorly understood.

Zolpidem is a short-acting non-benzodiazepine agent from the imidazopyridine class usually used against insomnia (Langtry and Benfield, 1990; Sanger, 2004). It has been shown to induce paradoxical responses in some patients with disorders of consciousness (DOC), leading to an improvement of arousal and cognitive abilities. Several case-studies showed that it can induce very impressive recoveries in severely brain-damaged patients with DOC of various etiologies (Clauss et al., 2000; Cohen et al., 2004; Clauss and Nel, 2006; Brefel-Courbon et al., 2007; Shames and Ring, 2008; Williams et al., 2013). However, this effect remains rare (i.e., around 5-7\% of responders; Whyte and 
Myers, 2009; Thonnard et al., 2013; Whyte et al., 2014) and it is not always significant in terms of functional recovery (in the $7 \%$ of the patients showing improvement, no diagnosis changes was observed in Thonnard et al., 2013). A change in brain activity (e.g., prefrontal cortices, thalami and striatum) after zolpidem intake has been reported in single subject studies using singlephoton emission computed tomography (measuring blood flow) (Clauss et al., 2000), positron emission tomography (PET, BrefelCourbon et al., 2007; Williams et al., 2013; measuring blood flow and metabolism), and electroencephalography (EEG; measuring electrical activity Williams et al., 2013).

We here assessed zolpidem-induced changes in regional brain metabolism in a case-series of three patients with known zolpidem response after chronic post-anoxic MCS. According to the mesocircuit model for the recovery of consciousness (Schiff, 2010), zolpidem is suggested to disinhibit the globus pallidus interna (GPi) and by that way increase the thalamic excitatory role on prefrontal cortices (see Figure 1). Based on this model, we hypothesized that an impaired brain metabolism in the thalamus, striatum and prefrontal areas would be observed at the group level during placebo, which would recover following zolpidem intake.

\section{MATERIALS AND METHODS}

Three patients with chronic post-anoxic MCS with known clinical improvement to zolpidem administration (i.e., EMCS) were included in the study. All patients took zolpidem on a regular basis for at least 6 months. For the present study, zolpidem intake was stopped at least $12 \mathrm{~h}$ prior to the research protocol. $10 \mathrm{mg}$ of zolpidem or placebo (water) were administered at 12 a.m. via gastrostomy in a randomized order, in a double blind 2-day design. All other treatments remained unchanged throughout the study. Standardized clinical assessment using the CRS-R (Giacino et al., 2004; Schnakers et al., 2008b) was performed $30 \mathrm{~min}$ after administration of zolpidem or placebo. The CRS-R is a validated and sensitive behavioral assessment scale to determine patients' level of consciousness (Seel et al., 2010). It assesses auditory, visual, verbal, and motor functions as well as communication and arousal level. The total score ranges between 0 (coma) and 23 (EMCS).

FDG-PET cerebral metabolism data were acquired $90 \mathrm{~min}$ after zolpidem or placebo intake, at the University Hospital of Liège using a Gemini TF Big Bore (Philips Medical System) and according to a standard clinical protocol. An intravenous injection of $300 \mathrm{MBq}$ fluorodeoxyglucose was administered $30 \mathrm{~min}$ before the FDG-PET. Patients were monitored by an anesthesiologist throughout the procedure. The study was approved by the Ethics Committee of the Faculty of Medicine of the University of Liège and written informed consent was obtained from the patients' legal representatives and all volunteers.

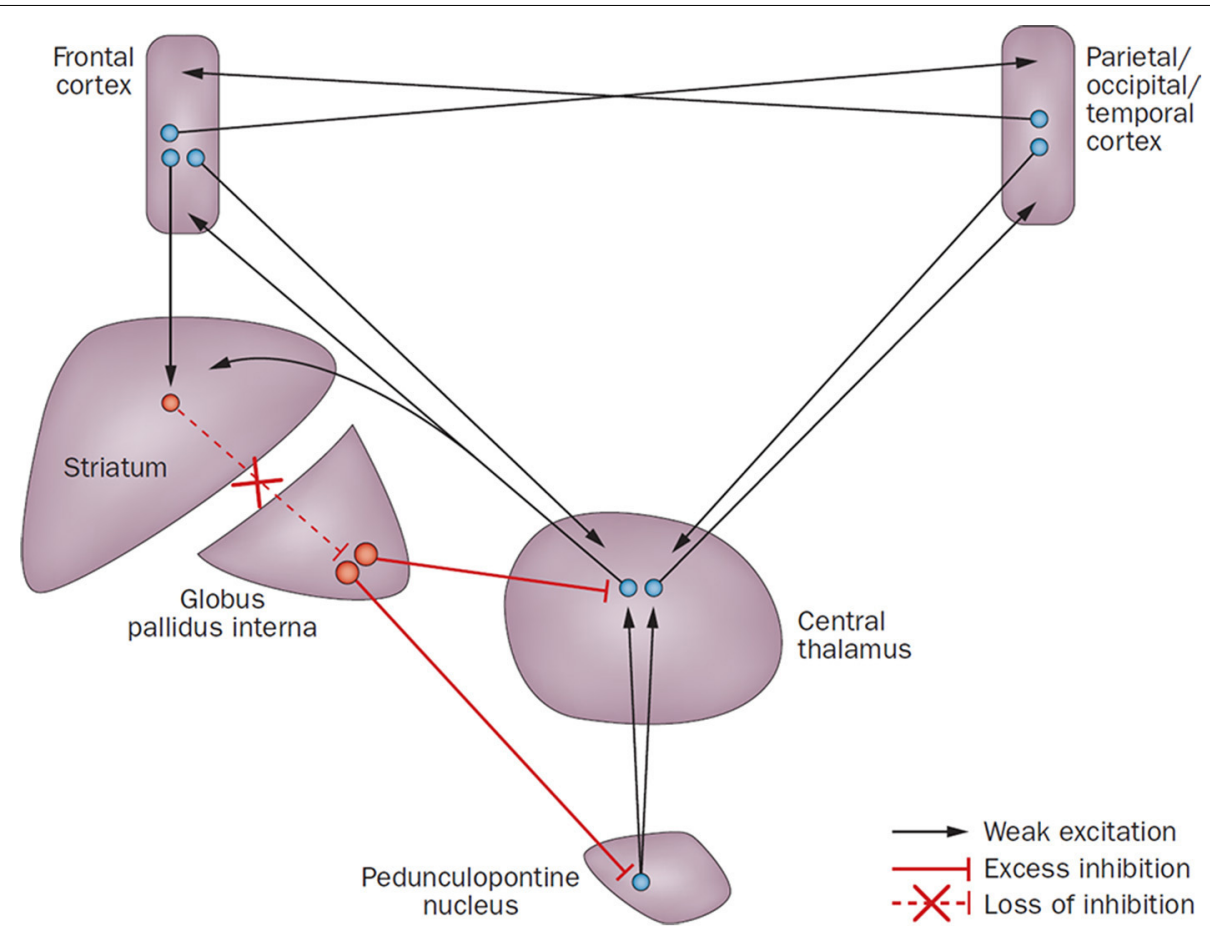

FIGURE 1 | The mesocircuit model underlying forebrain dysfunction and interventions in severe brain injuries (From Giacino et al., 2014, with permission). Reduction of thalamocortical and thalamostriatal outflow following deafferentation and neuronal loss from the central thalamus withdraws the afferent drive to the striatum, which may then fail to reach firing threshold because of their requirement for high levels of synaptic background activity. Loss of active inhibition from the striatum allows neurons of the globus pallidus interna to tonically fire and provide active inhibition to their synaptic targets, including relay neurons of the already disfacilitated central thalamus, and possibly also the projection neurons of the pedunculopontine nucleus. Since the GABAA a-1 subunit is normally expressed in large quantities in the globus pallidus interna, zolpidem could inhibit the latter, substituting its normal inhibition from the striatum, and hence induce an increase of the thalamic excitatory influence on prefrontal cortices. 
PET data of patients were compared to an age-matched group of 39 healthy participants (mean age $45 \pm 16$ years; 18 men). Preprocessing of the PET data was identical as previously published (Phillips et al., 2011; Bruno et al., 2012; Thibaut et al., 2012) including spatial normalization and smoothing (using a $14 \mathrm{~mm}$ full width at a half maximum Gaussian kernel), implemented in Statistical Parametric Mapping toolbox (SPM8; www.fil.ion.ucl. ac.uk/spm).

The statistical analyses were also performed using SPM8 toolbox. A full-factorial design with three design matrices modeled the subject-effect (MCS patient 1, 2, 3), drug-effect (placebo vs. zolpidem) and group effect (patients vs. controls) was performed. After proportional scaling, we identified brain regions that showed a relative increase in metabolism after zolpidem intake as compared to placebo. Areas showing an impaired metabolism after placebo and after zolpidem intake as compared to healthy controls were also investigated. Results were considered significant at false discovery rate cluster level $p<0.001$ after a Bonferroni correction for multiple comparisons.

\section{CASE REPORTS}

Table 1 shows the CRS-R subscores after placebo and zolpidem intake.

\section{MCS 1}

A 37-year-old female was assessed 18 months post anoxia (cardiorespiratory arrest after hanging). Within the month post-insult, she emerged from the vegetative state/unresponsive wakefulness

Table 1 | Behavioral assessments after placebo and zolpidem intake (based on the Coma Recovery Scale-Revised).

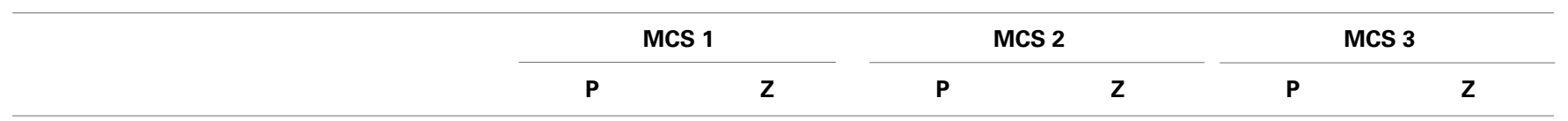

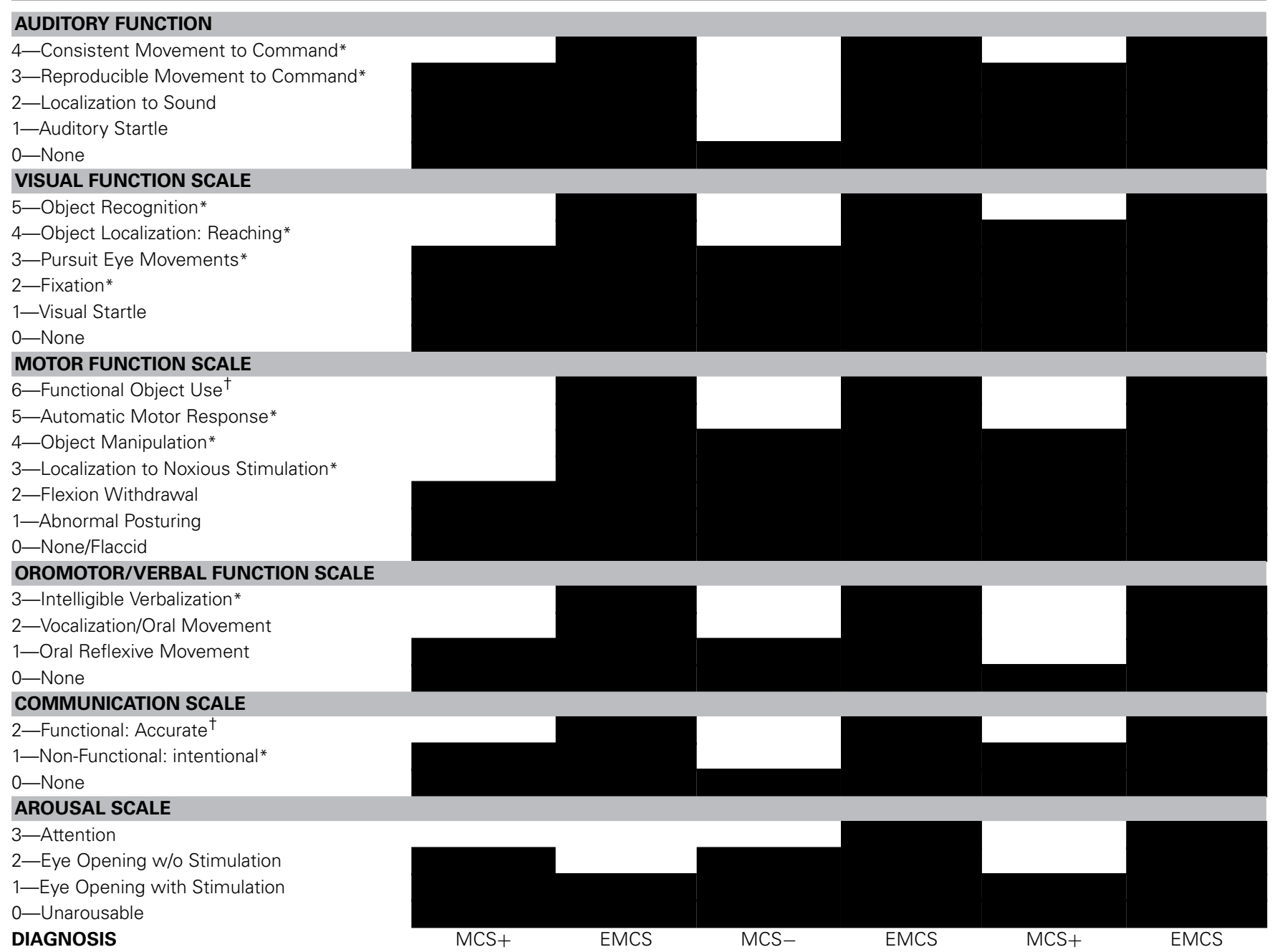

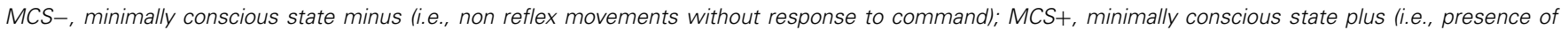
response to command); EMCS, emergence from the minimally conscious state (i.e., functional communication or object use).

* Indicates clinical signs compatible with MCS. 'Indicates emergence from minimally conscious state. P, placebo; Z, zolpidem. Highest score achieved for each subscale is represented by the black boxes. 
syndrome and evolved into a MCS plus. CRS-R assessment showed reproducible but not consistent command following, visual pursuit, and intentional communication. MRI showed ischemic brain lesions in the basal ganglia, in the left occipital and bilateral posterior parietal cortices. Resting EEG showed a reactive background rhythm of alpha/theta activity $(7-8 \mathrm{~Hz})$ without any epileptic activity. After zolpidem administration, she could systematically follow simple commands, recognize different objects and use them adequately, and communicate functionally (i.e., EMCS). Concurrent treatment consisted of amantadine, fluoxetine, trihexyphenidyl, tramadol, and esomeprazole.

\section{MCS 2}

A 38-year-old male was assessed 12 years and 7 months postanoxia (cardio-respiratory arrest after hanging). The patient showed visual pursuit and automatic motor reactions (i.e., mouth opening when a spoon was brought to his mouth), and was diagnosed as MCS minus. The MRI showed lesions in the brainstem and in the thalami with diffuse periventricular white matter damage, more pronounced in posterior regions and in the left hemisphere. Resting EEG showed a background rhythm of $7 \mathrm{~Hz}$ theta activity without epileptic activity. After zolpidem administration, he was able to systematically follow simple commands, recognize objects and use them consistently, and communicate functionally (i.e., EMCS). Concurrent treatment consisted of levetiracetam.

\section{MCS 3}

A 50-year-old female was assessed 7 years post anoxia (cardiorespiratory arrest after hanging). Structural MRI did not show focal abnormalities (PET glucose and activation blood-flow data have been reported elsewhere, Brefel-Courbon et al., 2007). She remained 3 weeks in a coma before recovering signs of consciousness. CRS-R assessment showed automatic motor reactions (i.e., pulling on her shirt), reproducible but not consistent command following, localization of objects, and intentional communication (i.e., MCS plus). Resting EEG recordings did not demonstrate any epileptic discharges with a background rhythm of $6 \mathrm{~Hz}$ theta activity. Following zolpidem intake, she showed consistent command following, functional use of objects, and functional communication (i.e., EMCS). Concurrent treatment consisted of modafinil, fluoxetine, piribedil, dihydroergocristine, domperidone, vinburnine, heptaminol, and omeprazole.

\section{RESULTS}

Group level analysis showed a relative increase in the bilateral superior frontal gyri and the right medial frontal cortex following zolpidem intake as compared to placebo (Table 2 and Figures 2, 3). At a less conservative statistical threshold uncorrected for multiple comparisons, the left insula, the middle frontal gyri and the left inferior frontal and parietal areas also showed a relative increase following zolpidem intake as compared with placebo. During placebo, the thalami and the left precuneus/posterior cingulate areas showed an impaired brain metabolism as compared with healthy controls. At a less conservative statistical threshold, bilateral superior and middle frontal gyri, the left precuneus/posterior cingulate, bilateral precentral gyri, left insula and right inferior parietal areas showed an impaired brain metabolism. After zolpidem intake, the thalami and the left precuneus/posterior cingulate areas showed an impaired brain metabolism as compared with healthy controls. At a less conservative threshold, the precentral gyri, left superior frontal and temporal gyri, the left middle frontal gyrus and precuneus and the right inferior parietal lobe showed an impaired brain metabolism.

\section{DISCUSSION}

Using a double-blind placebo-controlled design, we here report FDG-PET changes after the administration of zolpidem in three chronic post-anoxic patients in MCS who show clinically significant paradoxical behavioral improvements (i.e., EMCS). We observed that zolpidem-related recovery of cognitive abilities was paralleled by an increase in glucose metabolism in bilateral dorsolateral prefrontal and mesiofrontal cortices. Our findings support previous case-studies reporting a change in prefrontal cortex activity after zolpidem intake using single-photon emission computed tomography (Clauss et al., 2000), PET (Brefel-Courbon et al., 2007; Williams et al., 2013) and EEG (Williams et al., 2013). Prefrontal areas are known to be involved in the "limbic loop" regulation of motivation and are a key center of the mesocircuit model for consciousness (Schiff, 2010; Laureys and Schiff, 2012). The mesocircuit hypothesis for the effect of zolpidem (Schiff, 2010) supports the idea that, in normal cognitive processing, the striatum disinhibits the central thalamus via the internal globus pallidus (GPi) while the central thalamus promotes activity of prefrontal cortices. Therefore, if the activity in the striatum is reduced (e.g., following a severe brain injury), central thalamic and prefrontal activities are also reduced, possibly explaining the observed hypometabolism of the latter in the here reported patients in the baseline condition (i.e., after placebo administration). Since the GABAA a-1 subunit is normally expressed in large quantities in the GPi, zolpidem could inhibit the GPi, substituting the normal inhibition of the GPi from the striatum. The mesocircuit model assumes that zolpidem directly inhibits the GPi, substituting the normal inhibition of the GPi from the striatum (one of the most sensitive areas to cerebral hypoxia Calabresi et al., 2000) and would hence increase the thalamic excitatory influence on prefrontal cortices. Interestingly, in all studies with zolpidem responders (Clauss et al., 2000; BrefelCourbon et al., 2007), including ours, the brain areas showing increased metabolism after zolpidem did not show significant structural lesions. This could support the idea that patients who respond to zolpidem have neurologic deficits mainly caused by inhibitory functional effects rather than by severely structurally damaged or dead brain tissue (Shames and Ring, 2008). This effect is defined as cerebral diashisis, i.e., the loss of function in a portion of the brain (here, the prefrontal cortices) as a result of its connection to another injured area (here, striatum and/or thalami) (Glassman, 1971; Feeney and Baron, 1986; Tecco et al., 1998; Witte et al., 2000). It has been proposed that zolpidem may be effective to restore brain function in patients whose brain injuries are mainly located outside of brainstem structures (Du et al., 2014). However, in our three responders one 
Table 2 | Regions showing significant impaired metabolism following placebo and zolpidem and relative increase following zolpidem intake.

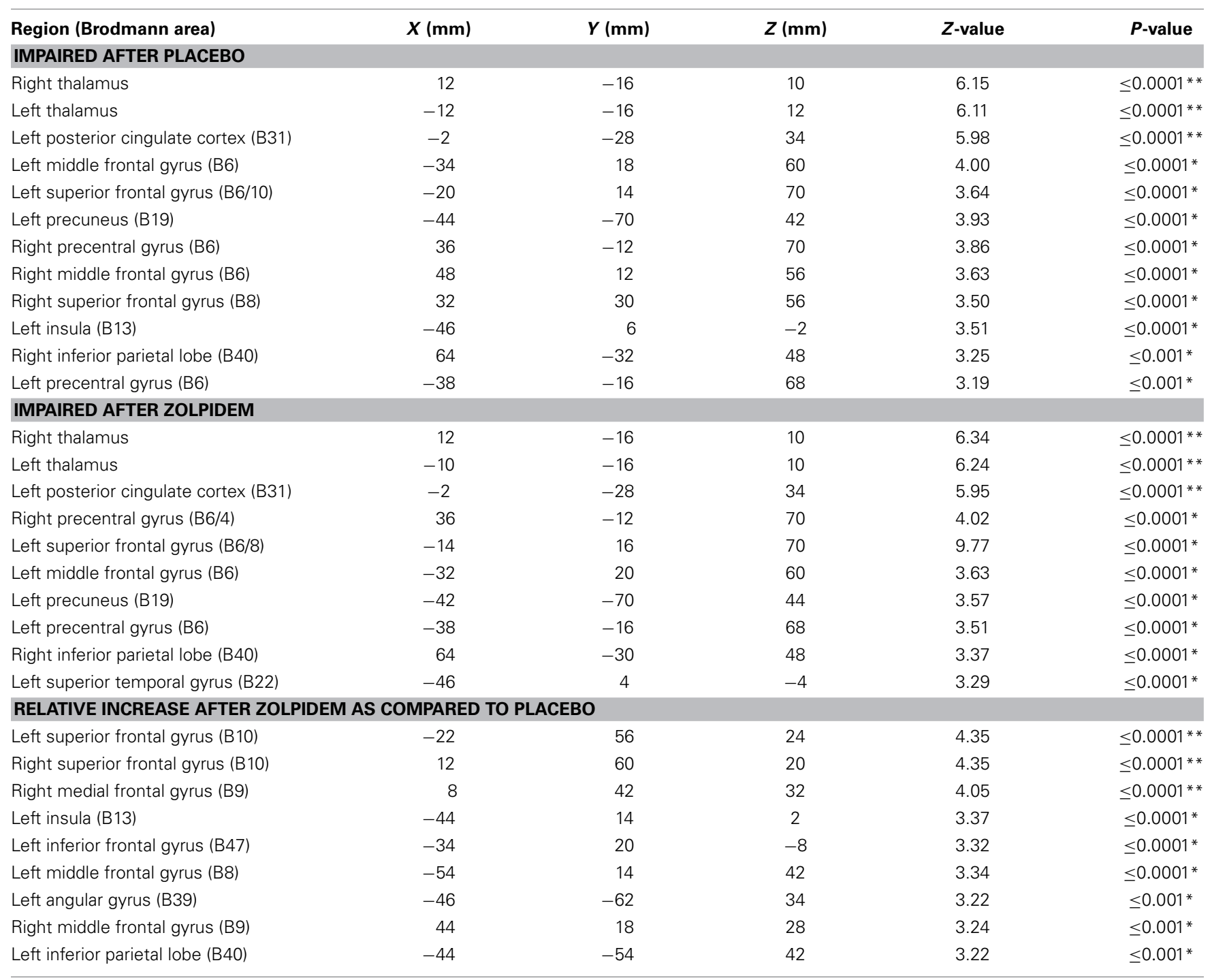

coordinates of peak voxels in standardized stereotaxic MNI space.

** Corrected p-value for multiple comparisons.

* Uncorrected p-value.

did show structural brainstem lesions (Du et al., 2013). In contrast to two previous case-studies (Brefel-Courbon et al., 2007; Williams et al., 2013), we did not observe a zolpidem-related increase in metabolism in thalamic and striatal regions. This could be explained either by the fact that the technique used is not sensitive enough to highlight small functional activity changes in these areas, or because zolpidem induced changes might modify effective connectivity between areas which cannot be investigated using FDG-PET. Future neuroimaging studies looking at effective connectivity in DOC zolpidem-responders would allow to better document the role of thalamo-cortico excitatory pathways underlying the observed increased activity in prefrontal areas. In addition, further studies focusing on regional glucose metabolism changes at the individual level in specific areas involved in the mesocircuit would help to better understand the mechanisms of recovery following zolpidem intake (Fridman et al., 2014).
Another theory, the "GABA impairment hypothesis" was recently proposed by Pistoia et al. (2014) to explain the effect of zolpidem on recovery of consciousness. This theory suggests that zolpidem (as well as baclofen, a GABA-B agonist) may act on consciousness recovery thanks to the restoration of a normal ratio between synaptic excitation and inhibition by reversing the impairment of GABA in this population of severely braininjured patients. This hypothesis has the advantage to explain the potential mode of action of both zolpidem and baclofen on patient's recovery. In some patients with an impaired balance of cortical subcortical-cortical connections, the use of GABA agonists would decrease excessive information and thus reorganize an equilibrate dialogue among different brain nuclei and allow proper information processing. Nevertheless, the time course of the observed effect of these two drugs is different. For zolpidem, the short-term effect that disappear when the plasma drug 

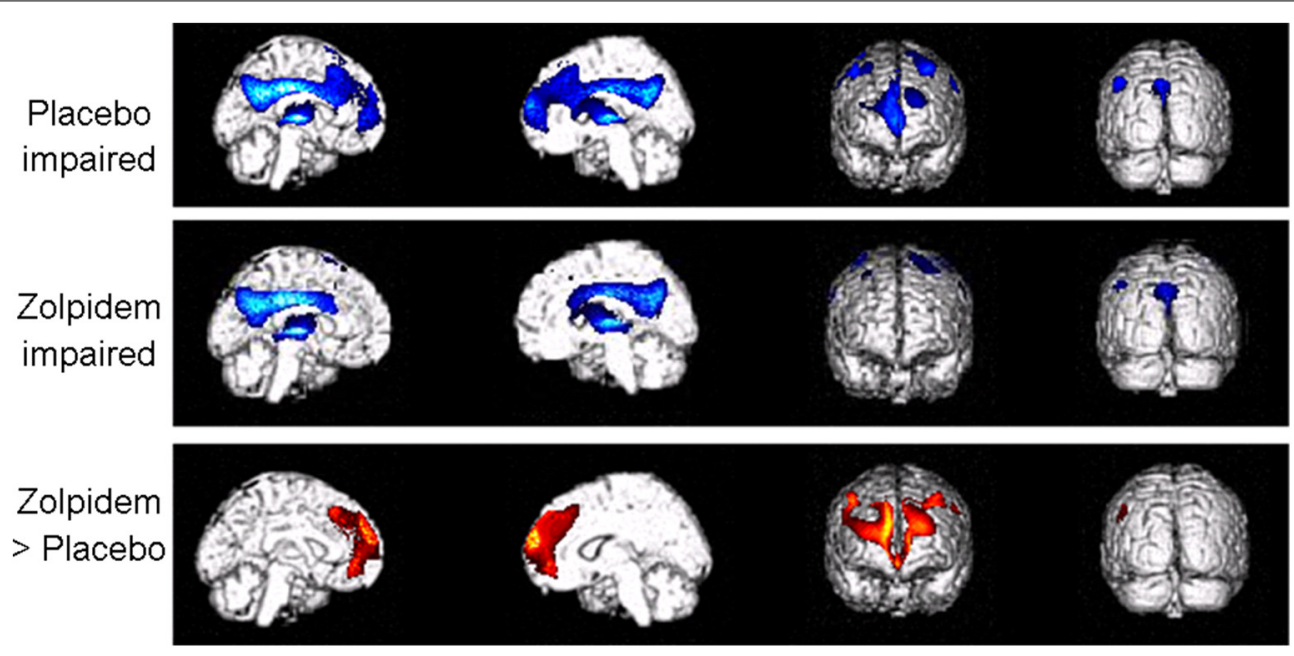

FIGURE 2 | Impaired brain metabolism after placebo and zolpidem intake and areas showing relative recovery after zolpidem. Brain areas showing impaired metabolism (in blue) following placebo and zolpidem administration and regions which were impaired following placebo but showed relative recovery of activity after zolpidem intake (in red). For display purposes results are shown thresholded at uncorrected $p<0.001$. From left to right, medial right and left view, frontal and posterior view of a $3 \mathrm{D}$ rendered brain $\mathrm{MRI}$.

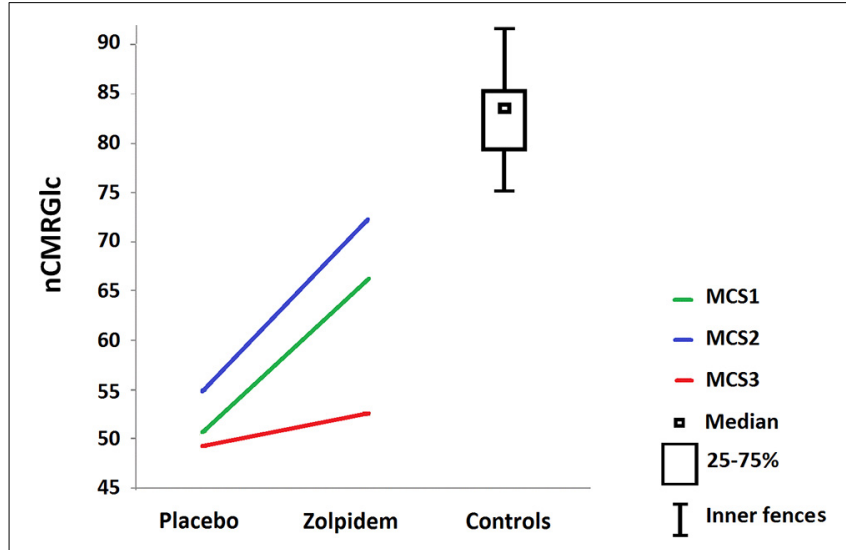

FIGURE 3 | Normalized cerebral metabolic rate for glucose (nCMRGIc) values in prefrontal cortices after placebo. Values reported for the cluster using peak coordinates $x, y, z=-22,56,24$ after placebo and zolpidem intake in patients, as compared to healthy controls (boxplot showing median, 25-75\% quartiles and inner fences).

concentration falls suggest rapid neurotransmitter changes. On the other hand, for baclofen, its slow-onset effects suggest a phenomenon of gradual neuroplasticity. Unfortunately, we cannot verify this hypothesis with the method used in our study (i.e., FDG-PET) this will therefore need to be addressed in future studies.

Finally, if all these theories can explain part of the mechanisms underlying the paradoxical responses induced by zolpidem, they do not explain why only less than $10 \%$ of the patients react positively to those GABA agonist drugs. Large cohort studies may help investigating this question.

We have to highlight that our study included only three patients, and all had a hypoxia; therefore, our results are not generalizable to the general DOC population (Adams et al., 2000).
Note also that not all the patients with post-anoxic DOC may improve after zolpidem. In addition, the three patients were very likely to suffer from depression prior to injury (as they all attempted suicide), which may be a confounding factor. One could argue that vigilance in MCS patients is fluctuating and the behavioral improvement observed in the three patients could be related to this bias. Nevertheless, these patients were in a chronic stage (i.e., more stable in their responses) and they showed a dramatic recovery of functional communication/object use that has not been observed before. These elements support the idea of a diagnostic change induced by zolpidem intake.

Our results also underline a key role of the prefrontal cortices in the recovery of functional communication and object use in those hypoxic patients with chronic DOC. It is well known that the prefrontal cortex has a major role in executive function and working memory, a recovery of its functionality is therefore likely to influence object use and recovery of communication. Our findings partly corroborate previous case-studies (Nakayama et al., 2006; Thibaut et al., 2012) reporting a decreased metabolism in medial prefrontal and frontobasal regions, cingulate gyrus and thalamus as a function of the consciousness impairments in severely brain-injured patients. In addition, we observed a relative decrease in brain metabolism in the left superior temporal gyrus following zolpidem intake. This could be explained by the reestablishment of top-down processes associated with the reactivation of the prefrontal areas following zolpidem.

Taken together, the data suggest that the infrequent but existing paradoxical effect of zolpidem could be characteristic to patients having suffered subcortical thalamic (as in all 3 cases here reported) and/or striatal functional lesions preventing prefrontal cortices to exhibit their normal function.

\section{CONCLUSION}

We observed an increased metabolism in prefrontal cortices following zolpidem intake, in line with the previously proposed 
mesocircuit model for recovery of consciousness in DOC (Schiff, 2010). Large cohort studies comparing brain structure, function and mesocircuit connectivity of DOC zolpidem-responders as compared to the vast majority of non-responders would allow to better understand in which cases zolpidem might improve cognitive function in such a clinically significant and yet transient manner. Increasing our research efforts to understand the common denominator and neural mechanisms underlying these rare cases responding to zolpidem could provide new avenues for promoting therapeutic strategies improving the recovery of some DOC patients with severe acquired brain damage.

\section{AUTHOR CONTRIBUTIONS}

Camille Chatelle designed the study, performed the statistical analysis and drafted the manuscript with Aurore Thibaut. Olivia Gosseries participated in medical data collection and helped drafting the manuscript. Mohamed A. Bahri participated in the design of the study and acquired behavioral and PET data. Athena Demertzi collected behavioral and MRI data. Claire Bernard and Roland Hustinx performed PET examination and preprocessed the data. Luaba Tshibanda did the visual analysis of structural MRI data. Marie-Aurélie Bruno revised the manuscript critically for important methodological content. Steven Laureys participated in the design of the study and revised the manuscript critically for important intellectual content. All authors read, commented and approved the final manuscript.

\section{ACKNOWLEDGMENTS}

This study was supported by the National Funds for Scientific Research (FNRS), Action de Recherche Concertée, Fonds Léon Fredericq, James S. McDonnell Foundation, Mind Science Foundation, University of Liège, the Belgian American Educational Foundation (BAEF), the Fédération Wallonie Bruxelles International (WBI) and the Belgian interuniversity attraction pole. Camille Chatelle and Olivia Gosseries are funded by the BAEF and WBI; Olivia Gosseries, Mohamed A. Bahri and Athena Demertzi are FNRS post-doctoral researchers and Steven Laureys is FNRS research director.

\section{REFERENCES}

Adams, J. H., Graham, D. I., and Jennett, B. (2000). The neuropathology of the vegetative state after an acute brain insult. Brain 123(Pt 7), 1327-1338. doi: 10.1093/brain/123.7.1327

Brefel-Courbon, C., Payoux, P., Ory, F., Sommet, A., Slaoui, T., Raboyeau, G., et al. (2007). Clinical and imaging evidence of zolpidem effect in hypoxic encephalopathy. Ann. Neurol. 62, 102-105. doi: 10.1002/ana.21110

Bruno, M. A., Majerus, S., Boly, M., Vanhaudenhuyse, A., Schnakers, C., Gosseries, O., et al. (2012). Functional neuroanatomy underlying the clinical subcategorization of minimally conscious state patients. J. Neurol. 259, 1087-1098. doi: 10.1007/s00415-011-6303-7

Bruno, M. A., Vanhaudenhuyse, A., Thibaut, A., Moonen, G., and Laureys, S. (2011). From unresponsive wakefulness to minimally conscious PLUS and functional locked-in syndromes: recent advances in our understanding of disorders of consciousness. J. Neurol. 258, 1373-1384. doi: 10.1007/s00415-0116114-x

Calabresi, P., Centonze, D., and Bernardi, G. (2000). Cellular factors controlling neuronal vulnerability in the brain: a lesson from the striatum. Neurology 55, 1249-1255. doi: 10.1212/WNL.55.9.1249

Clauss, R., and Nel, W. (2006). Drug induced arousal from the permanent vegetative state. NeuroRehabilitation 21, 23-28.
Clauss, R. P., Guldenpfennig, W. M., Nel, H. W., Sathekge, M. M., and Venkannagari, R. R. (2000). Extraordinary arousal from semi-comatose state on zolpidem. A case report. S. Afr. Med. J. 90, 68-72.

Cohen, L., Chaaban, B., and Habert, M. O. (2004). Transient improvement of aphasia with zolpidem. N. Engl. J. Med. 350, 949-950. doi: 10.1056/NEJM200402263500922

Du, B, Shan, A, Zhang, Y, Zhong, X, Chen, D, and Cai, K. (2013). Zolpidem arouses patients in vegetative state after brain injury: quantitative evaluation and indications. Am. J. Med. Sci. 347, 178-182. doi: 10.1097/MAJ.0b013e318287c79c

Du, B., Shan, A., Zhang, Y., Zhong, X., Chen, D., and Cai, K. (2014). Zolpidem arouses patients in vegetative state after brain injury: quantitative evaluation and indications. Am. J. Med. Sci. 347, 178-182. doi: 10.1097/MAJ.0b013e318287c79c

Feeney, D. M., and Baron, J. C. (1986). Diaschisis. Stroke 17, 817-830. doi: 10.1161/01.STR.17.5.817

Fridman, E. A., Beattie, B. J., Broft, A., Laureys, S., and Schiff, N. D. (2014). Regional cerebral metabolic patterns demonstrate the role of anterior forebrain mesocircuit dysfunction in the severely injured brain. Proc. Natl. Acad. Sci. U.S.A. 111, 6473-6478. doi: 10.1073/pnas.1320969111

Fridman, E. A., Calvar, J., Bonetto, M., Gamzu, E., Krimchansky, B. Z., Meli, F., et al. (2009). Fast awakening from minimally conscious state with apomorphine. Brain Inj. 23, 172-177. doi: 10.1080/02699050802649662

Giacino, J., Ashwal, S., Childs, N., Cranford, R., Jennett, B., Katz, D., et al. (2002). The minimally conscious state: definition and diagnostic criteria. Neurology 58 , 349-353. doi: 10.1212/WNL.58.3.349

Giacino, J., Kalmar, K., and Whyte, J. (2004). The JFK Coma Recovery ScaleRevised: measurement characteristics and diagnostic utility. Arch. Phys. Med. Rehabil. 85, 2020-2029. doi: 10.1016/j.apmr.2004.02.033

Giacino, J. T., Fins, J. J., Laureys, S., and Schiff, N. D. (2014). Disorders of consciousness after acquired brain injury: the state of the science. Nat. Rev. Neurol. 10, 99-114. doi: 10.1038/nrneurol.2013.279

Giacino, J. T., Whyte, J., Bagiella, E., Kalmar, K., Childs, N., Khademi, A., et al. (2012). Placebo-controlled trial of amantadine for severe traumatic brain injury. N. Engl. J. Med. 366, 819-826. doi: 10.1056/NEJMoa1102609

Glassman, R. B. (1971). Recovery following sensorimotor cortical damage: evoked potentials, brain stimulation and motor control. Exp. Neurol. 33, 16-29. doi: 10.1016/0014-4886(71)90098-7

Gosseries, O., Charland-Verville, V., Thonnard, M., Bodart, O., Laureys, S., and Demertzi, A. (2013). Amantadine, apomorphine and zolpidem in the treatment of disorders of consciousness. Curr. Pharm. Des. 20, 4167-4184. doi: $10.2174 / 13816128113196660654$

Langtry, H. D., and Benfield, P. (1990). Zolpidem. A review of its pharmacodynamic and pharmacokinetic properties and therapeutic potential. Drugs 40, 291-313. doi: 10.2165/00003495-199040020-00008

Laureys, S., Celesia, G. G., Cohadon, F., Lavrijsen, J., Leon-Carrrion, J., Sannita, W. G., et al. (2010). Unresponsive wakefulness syndrome: a new name for the vegetative state or apallic syndrome. BMC Med. 8:68. doi: 10.1186/17417015-8-68

Laureys, S., and Schiff, N. D. (2012). Coma and consciousness: paradigms (re)framed by neuroimaging. Neuroimage 61, 478-491. doi: 10.1016/j.neuroimage.2011.12.041

Nakayama, N., Okumura, A., Shinoda, J., Nakashima, T., and Iwama, T. (2006). Relationship between regional cerebral metabolism and consciousness disturbance in traumatic diffuse brain injury without large focal lesions: an FDGPET study with statistical parametric mapping analysis. J. Neurol. Neurosurg. Psychiatry 77, 856-862. doi: 10.1136/jnnp.2005.080523

Phillips, C. L., Bruno, M. A., Maquet, P., Boly, M., Noirhomme, Q., Schnakers, C., et al. (2011). "Relevance vector machine" consciousness classifier applied to cerebral metabolism of vegetative and locked-in patients. Neuroimage 56, 797-808. doi: 10.1016/j.neuroimage.2010.05.083

Pistoia, F., Sara, M., Sacco, S., Franceschini, M., and Carolei, A. (2014). Silencing the brain may be better than stimulating it. The GABA effect. Curr. Pharm. Des. 20, 4154-4166.

Sanger, D. J. (2004). The pharmacology and mechanisms of action of new generation, non-benzodiazepine hypnotic agents. CNS Drugs 18(Suppl. 1:9-15; discussion 41), 3-5.

Sara, M., Sacco, S., Cipolla, F., Onorati, P., Scoppetta, C., Albertini, G., et al. (2007). An unexpected recovery from permanent vegetative state. Brain Inj. 21, 101-103. doi: 10.1080/02699050601151761 
Schiff, N. D. (2010). Recovery of consciousness after brain injury: a mesocircuit hypothesis. Trends Neurosci. 33, 1-9. doi: 10.1016/j.tins.2009.11.002

Schiff, N. D., Giacino, J. T., Kalmar, K., Victor, J. D., Baker, K., Gerber, M., et al. (2007). Behavioural improvements with thalamic stimulation after severe traumatic brain injury. Nature 448, 600-603. doi: 10.1038/nature06041

Schnakers, C., Hustinx, R., Vandewalle, G., Majerus, S., Moonen, G., Boly, M., et al. (2008a). Measuring the effect of amantadine in chronic anoxic minimally conscious state. J. Neurol. Neurosurg. Psychiatry 79, 225-227. doi: 10.1136/jnnp.2007.124099

Schnakers, C., Majerus, S., Giacino, J., Vanhaudenhuyse, A., Bruno, M., Boly, M., et al. (2008b). A French validation study of the Coma Recovery Scale-Revised (CRS-R). Brain Inj. 22, 786-792. doi: 10.1080/02699050802403557

Seel, R. T., Sherer, M., Whyte, J., Katz, D. I., Giacino, J. T., Rosenbaum, A. M., et al. (2010). Assessment scales for disorders of consciousness: evidence-based recommendations for clinical practice and research. Arch. Phys. Med. Rehabil. 91, 1795-1813. doi: 10.1016/j.apmr.2010.07.218

Shames, J. L., and Ring, H. (2008). Transient reversal of anoxic brain injury-related minimally conscious state after zolpidem administration: a case report. Arch. Phys. Med. Rehabil. 89, 386-388. doi: 10.1016/j.apmr.2007.08.137

Tecco, J. M., Wuilmart, P., Lasseaux, L., Wikler, D. Jr., Damhaut, P., Goldman, S., et al. (1998). Cerebello-thalamo-cerebral diaschisis: a case report. J. Neuroimaging 8, 115-116.

Thibaut, A., Bruno, M. A., Chatelle, C., Gosseries, O., Vanhaudenhuyse, A., Demertzi, A., et al. (2012). Metabolic activity in external and internal awareness networks in severely brain-damaged patients. J. Rehabil. Med. 44, 487-494. doi: 10.2340/16501977-0940

Thonnard, M., Gosseries, O., Demertzi, A., Lugo, Z., Vanhaudenhuyse, A., MarieAurelie, B., et al. (2013). Effect of zolpidem in chronic disorders of consciousness: a prospective open-label study. Funct. Neurol. 28, 259-264. doi: 10.11138/FNeur/2013.28.4.259

Whyte, J., and Myers, R. (2009). Incidence of clinically significant responses to zolpidem among patients with disorders of consciousness: a prelimi- nary placebo controlled trial. Am. J. Phys. Med. Rehabil. 88, 410-418. doi: 10.1097/PHM.0b013e3181a0e3a0

Whyte, J., Rajan, R., Rosenbaum, A., Katz, D., Kalmar, K., Seel, R., et al. (2014). Zolpidem and restoration of consciousness. Am. J. Phys. Med. Rehabil. 93, 101-113. doi: 10.1097/PHM.0000000000000069

Williams, S. T., Conte, M. M., Goldfine, A. M., Noirhomme, Q., Gosseries, O., Thonnard, M., et al. (2013). Common resting brain dynamics indicate a possible mechanism underlying zolpidem response in severe brain injury. Elife 2:e01157. doi: 10.7554/eLife.01157

Witte, O. W., Bidmon, H. J., Schiene, K., Redecker, C., and Hagemann, G. (2000). Functional differentiation of multiple perilesional zones after focal cerebral ischemia. J. Cereb. Blood Flow Metab. 20, 1149-1165. doi: 10.1097/00004647200008000-00001

Conflict of Interest Statement: The authors declare that the research was conducted in the absence of any commercial or financial relationships that could be construed as a potential conflict of interest.

Received: 18 July 2014; accepted: 27 October 2014; published online: 02 December 2014.

Citation: Chatelle C, Thibaut A, Gosseries O, Bruno M-A, Demertzi A, Bernard C, Hustinx R, Tshibanda L, Bahri MA and Laureys S (2014) Changes in cerebral metabolism in patients with a minimally conscious state responding to zolpidem. Front. Hum. Neurosci. 8:917. doi: 10.3389/fnhum.2014.00917

This article was submitted to the journal Frontiers in Human Neuroscience.

Copyright (๑) 2014 Chatelle, Thibaut, Gosseries, Bruno, Demertzi, Bernard, Hustinx, Tshibanda, Bahri and Laureys. This is an open-access article distributed under the terms of the Creative Commons Attribution License (CC BY). The use, distribution or reproduction in other forums is permitted, provided the original author(s) or licensor are credited and that the original publication in this journal is cited, in accordance with accepted academic practice. No use, distribution or reproduction is permitted which does not comply with these terms. 